\title{
Design and Structural Analysis for an Autonomous UAV System Consisting of Slave MAVs with Obstacle Detection Capability Guided by a Master UAV Using Swarm Control
}

\author{
Lakshmi Narashiman Aswin ${ }^{1}$, Prasanth Rajasekaran ${ }^{2}$, \\ Santhosh Kumar Radhakrishnan ${ }^{3}$, K.Shivarama Krishnan ${ }^{4}$ \\ ${ }^{I}$ (Department Of Mechanical Engineering, College Of Engineering Guindy, India) \\ ${ }^{2}$ (Department Of Mechanical Engineering, College Of Engineering Guindy, India) \\ ${ }_{3}^{3}$ (Department Of Mechanical Engineering, College Of Engineering Guindy, India) \\ ${ }^{4}$ (Department Of Biotechnology, Sathyabama University, India)
}

\begin{abstract}
An Unmanned Aerial Vehicle (UAV) is an aircraft without a human pilot. It can either be controlled manually by a pilot on the ground using a trans-receiver or it can be programmed to operate autonomously. In this proposed control system, multiple slave Micro Aerial Vehicles(MAV) are dispatched from a master UAV for surveillance. All the MAVs are synchronized with each other through the master UAV which highlights their purpose and position. The master UAV acts as a mobile base for the surveillance, it stores the data collected by the MAVs and transmits them to a remote base. A design of the UAV-MAV system and its performance analysis is presented.
\end{abstract}

Keywords- Autonomous control, Characteristics, Linux, Master / Slave Aerial Vehicles, NX 8.0 Nastran, Surveillance.

\section{INTRODUCTION}

Unmanned Aerial Vehicles have become an integral part in various fields. They are used in situations in which actual presence of pilots may be unsafe due to dangerous or biologically hazardous environments. Unmanned Aerial Vehicles can be fit with a high resolution camera that can be used aerial photography or video recording. The aerial photography applications range from documentary of wildlife to military stealth surveillance applications. Image processing techniques can also be applied for the procured images as well as for object identification during surveillance missions. The video captured during flight can be directly streamed by the pilot, enabling him to remotely control the UAV thousands of meters away. UAVs can be completely automated using various sensors such as ultrasonic sensors to achieve autonomous motion with obstacle avoidance capabilities in very close spaces. Autonomous control can be applied in situations where there are blind spots and accurate manual control cannot be achieved. Micro Aerial Vehicles are used in search and rescue missions which are almost impossible to carry out manually with limited ground vision of the terrain and inability to locate or approach hurt civilians trapped under large chunks of debris or inaccessible paths. UAVs are utilized in 3-D image of environment and obtaining information such as temperature, pressure and moisture content in hazardous environments for research applications.

\section{MASTER / SLAVE CONTROL THEORY}

This paper introduces a novel concept of Master and Slave control theory and an insight into the involved intercommunication between several Unmanned Aerial Vehicles. This control theory is based on a model of communication between several electronic devices in which one device holds complete domination over the others. The latter device is known as the master while the other devices are known as slaves. In case of several Unmanned Aerial Vehicles, one UAV acts as a master while the others act as slaves. The slaves acquire information from the surrounding using sensors such as pressure, temperature and ultrasonic sensors and transmit the data to the centralized master UAV which processes the acquired data. The master UAV, after processing the data, sends the required instructions to individual slave UAVs to carry out their specific tasks. The slave UAVs are also capable of communicating with each other through the centralized master UAV enabling them to effectively split their actions to complete the required task in minimal time. The 'Master and Slave' control theory is very effective in completing complex tasks where proper synchronization between devices is required. The master UAV can also be linked with a ground station[1] ( e.g. laptop) through which all the input transferred to the master UAV from the slave MAVs. The user at the ground station can stream videos captured by video cameras attached to the slave MAVs by utilising the master UAV as a intermediate medium for communication. Therefore surveillance over a wide range of area can be established. 


\begin{tabular}{|c|c|c|}
\hline \multicolumn{3}{|c|}{ HARDWARE } \\
\hline Components & Specification & Quantity \\
\hline Raspberry pi & Model B & 2 \\
\hline Arduino Leonardo & - & 2 \\
\hline Arduino Nano & - & 4 \\
\hline Robotic OS & Linux C & - \\
\hline Infrared sensor & - & 14 \\
\hline Ultrasonic sensor & HC SR04, max 6m & 8 \\
\hline Gyroscopes & - & 5 \\
\hline Accelerometer & - & 5 \\
\hline Magnetometer & - & 5 \\
\hline Micro camera & $720 \mathrm{p} \mathrm{HD}, 30 \mathrm{fps}$ & 5 \\
\hline Battery & $6000 \mathrm{mAh}, 14.8 \mathrm{~V}$ & 2 \\
\hline \multirow[t]{2}{*}{ Brushless motors } & 810kv(UAV), 2100kv(MAV) & 4 \\
\hline & $50 \mathrm{~A}(\mathrm{UAV})$ & 8 \\
\hline Electronic speed & $10 \mathrm{~A}(\mathrm{MAV})$ & 4 \\
\hline controller & & 8 \\
\hline
\end{tabular}

\section{HARdWARE CONNECTIONS AND CONTROL CirCuIT}

Linux $\mathrm{C}$ is used as the operating system for controlling the actions of the master and slave quad rotor system. In the proposed system two Raspberry Pi (Model B) components, programmed and augmented with a Linux operating system[2], are placed on the frame of the master quad rotor and in the Ground Control location respectively. The Raspberry pi controllers in the master quad rotor and the Ground Control location are wirelessly connected. Instructions from the Ground Control location are directly transmitted to the master quad rotor using the Linux operating software and Raspberry pi controllers. The Raspberry pi microcontroller in the master quad rotor is connected to an Arduino Leonardo microcontroller. The Leonardo microcontroller mainly helps in stabilizing the quad rotor with the help of attached three axis Accelerometers, Gyroscopes and Magnetometers. The master quad rotor is designed to carry a satisfactory amount of payload and has very high processing power. The slave MAVs[3] are attached with Arduino Nano and connected wirelessly to the master quad rotor using a GSM or wireless shield. The master quad rotor and the slave MAVs are provided with obstacle avoidance capabilities by addition of ultrasonic sensors and infrared sensors[4][5].The data acquired by the MAVs during surveillance, such as images, are not only processed by their individual microcontrollers but also transmitted to the master UAV where secondary processing takes place. The acquired data are also transmitted to the user at the ground control station. The master UAV sends the necessary instructions to the slave MAVs based on the processed data

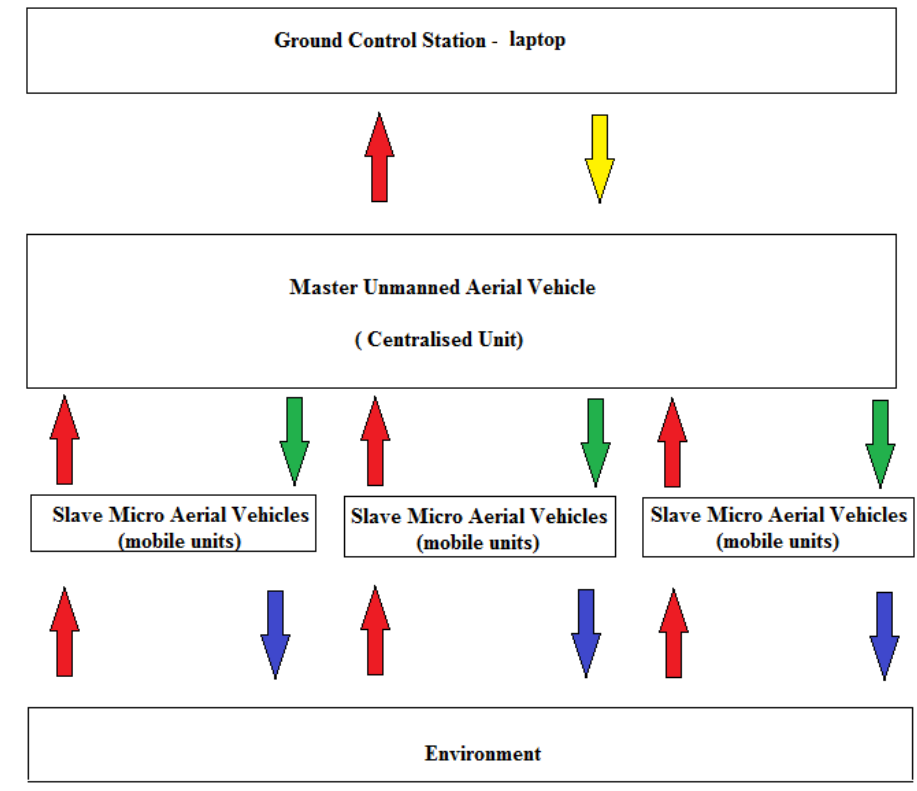

Fig. 1.Interaction between UAV-MAV system and surrounding.

As shown in the above Fig. 1, a longer distance and wider area can be covered by utilizing master and slave control theory to UAV systems. The Ground Control Station might not be able to directly communicate with 
slave MAVs with satellites at very long distances. However when master UAVs are used as a central medium for communication with other UAVs, surveillance missions can be carried out at much longer distances in a lesser time duration[6]. This system also offers another significant advantage. The MAVs do not have to process all the collected information individually. The information obtained by the MAVs can be directly transferred to the master UAV for processing owing to its high processing capability. The master UAV can also be augmented with extra microcontrollers to further improve its processing capabilities.

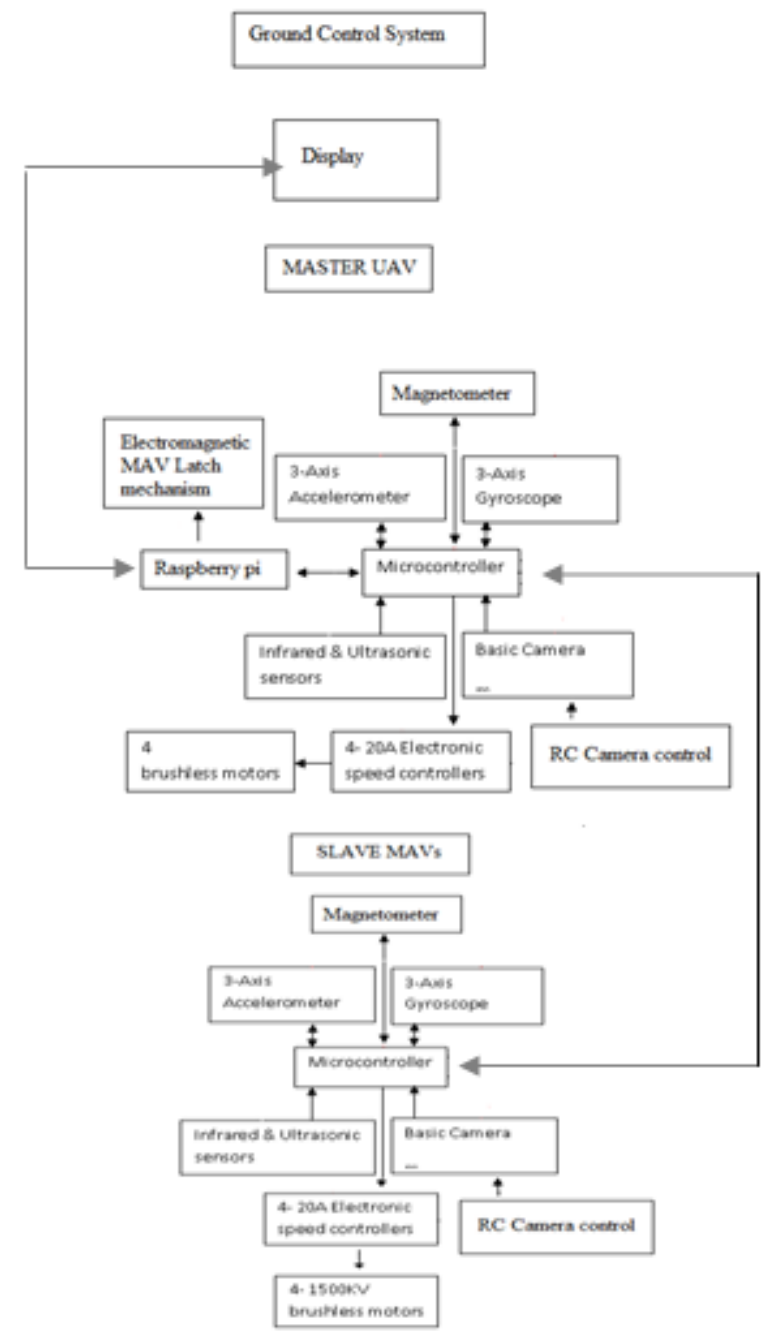

Fig. 2. Flowchart expressing the link between components.

\section{Performance AnAlysis Of MASTER /Slave SySTEM}

The analysis of the various factors influencing the performance of the UAV is presented. The source battery $(6000 \mathrm{mAh})$ provides a maximum voltage of $14.8 \mathrm{~V}$ at ideal conditions (w/o losses). This analysis takes into consideration the internal resistance $(\mathrm{R} 1 \approx 0.0322 \Omega)$ and thus, the voltage drops accordingly.

$$
V_{i}=V-I \times R_{1}
$$

I is the input current.

For various values of the input current and the given voltage, the Input power is given by:

$$
P_{i n}=V_{i} \times I
$$




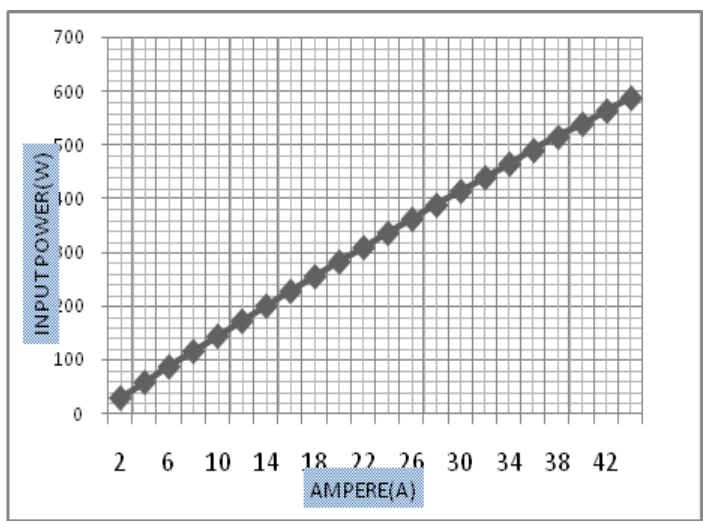

By consideration of the losses imparted by the motor resistance $(\mathrm{R} 2 \approx 0.052 \Omega)$, we get the corresponding Output power transmitted to the shaft of the motor as[7]:

$$
P_{\text {out }}=\left(V_{i}-I \times R_{2}\right) \times\left(I-I_{o}\right)
$$

Where, Io is the no-load current.

The efficiency is given by:

$$
\eta=P_{\text {out }} / P_{\text {in }} \%
$$

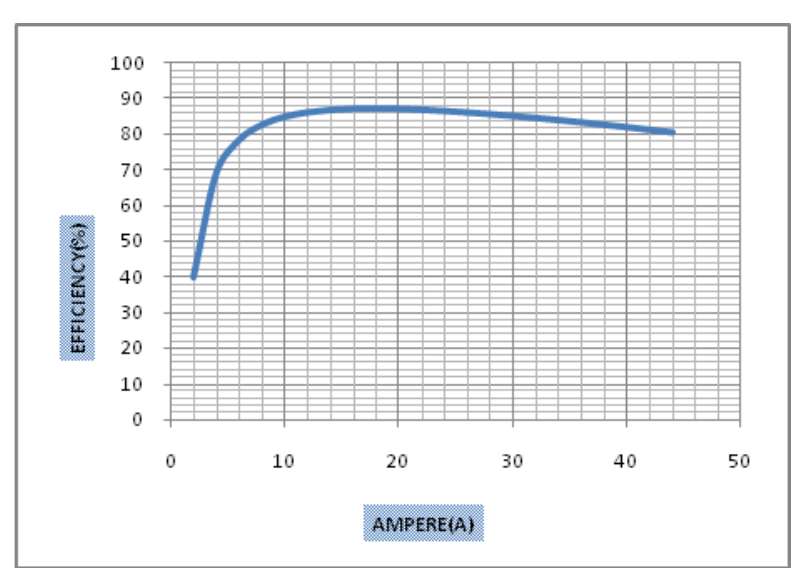

Fig. 4. Graph of Efficiency(\%) Vs. Current(Amperes)

The RPM achieved is given by[8]:

$$
R P M=K_{v} \times\left(V_{i}-I \times R_{2}\right)
$$

Where, $\mathrm{Kv}$ is the motor velocity constant

The thrust achieved by a single rotor[9]:

$$
T=\sqrt[s]{\left[\left(\mathrm{n}_{\text {prop }} \times P\right)^{2} \times 2 \pi \times R^{2} \rho\right]}
$$

Where, ${ }^{\eta_{\text {prop }}}=$ propeller hover efficiency $(=0.75)$

$\mathrm{P}=$ Output Power in Watts

$\mathrm{R}=$ propeller radius (metres)

$\rho=$ Density of air $(1.18 \mathrm{~kg} / \mathrm{m} 3)$ 




\section{BALANCING SHIFT IN CENTER OF GRAVITY}

When need arises for flight of particular pattern arrangement of MAV's such as flight of two adjacent MAV's, any three MAV's or a single MAV's, the Master UAV faces a shift in center of gravity from its center. This shift needs to be compensated for the UAV to sustain a stable flight (Fig. 6). The following formula[10] provides the distribution of the total thrust $\left(\mathrm{F}_{\text {Total }}\right)$ so as to maintain a stable flight at hover conditions.

$$
\begin{gathered}
F_{1}+F_{2}=F_{\text {Total }} \\
F_{2} / F_{1}=(L+X) /(L-X)
\end{gathered}
$$

Where, $F_{2}$ is Thrust value of the rotor close to the shift center of gravity while $F_{1}$ is the thrust value of the of rotor further away from the shifted center of gravity, $\mathrm{X}$ is the shift in center of gravity and $\mathrm{L}$ represents half the distance between the motors.

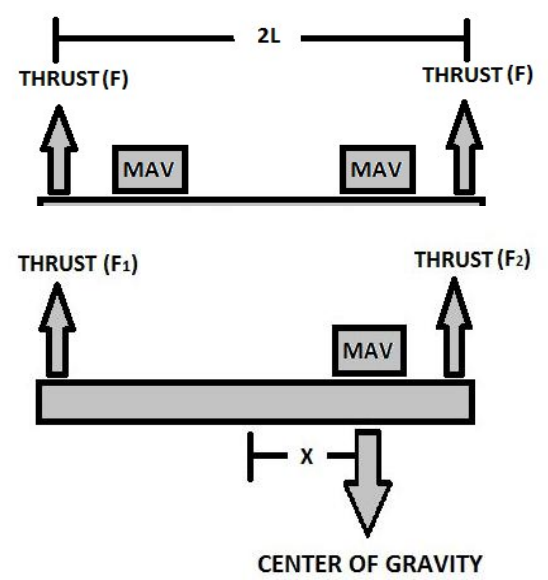

X-Shift in Center of Gravity

Fig. 6. Shift in Center of gravity

\section{Proposed STRUCTURAL DESIGn (PRO/ENGINEER WildFire 4.0) \\ 7.1 UAV-MAV System}






Fig. 7.Top view of UAV-MAV System

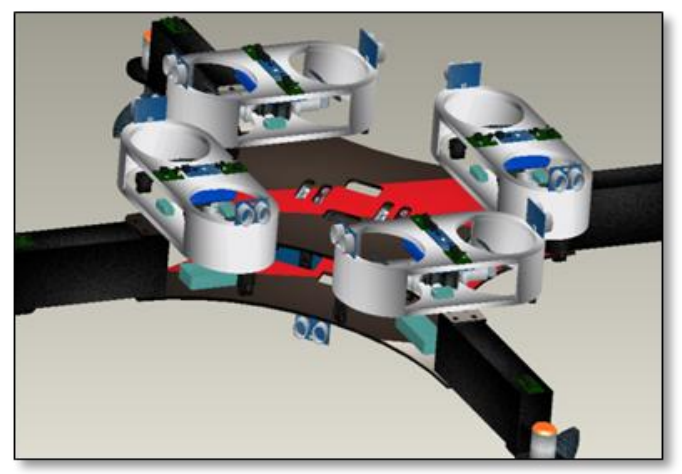

Fig. 8.Isometric view of UAV-MAV System

\subsection{Slave MAV (Bi copter)}

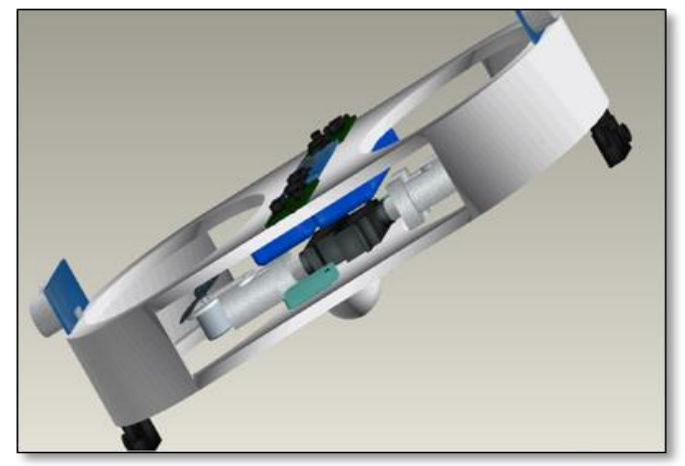

Fig. 9.The hemisphere at the bottom of the MAV frame is used for magnetic latching with the quad rotor frame.

\section{EXPLODED VIEW}

\subsection{Slave MAV (Bi Copter)}

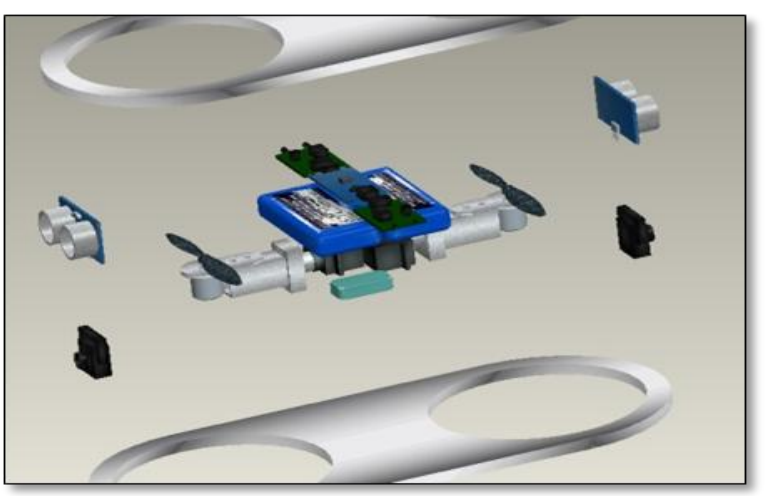

Fig. 10.Exploded view of Slave MAV 


\subsection{Master UAV (Quad Rotor)}

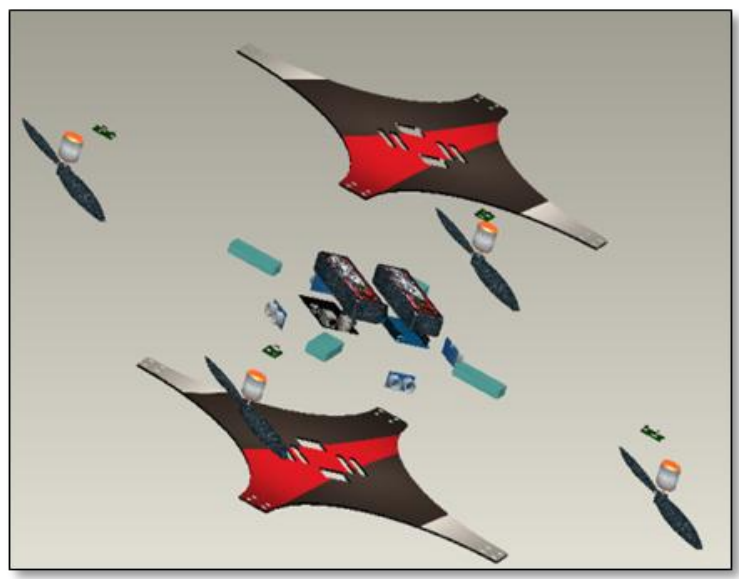

Fig. 11.Exploded view of Master UAV

\subsection{Assembled Model}

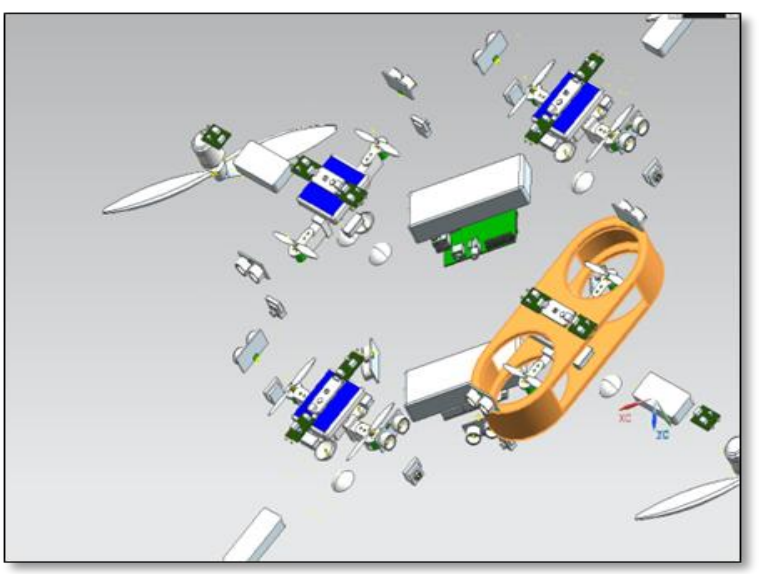

Fig. 12.Assembled view of UAV-MAV System

IX. Displacement, Stress And Reaction Force Analysis (During Take-Off)

The design of master and slave quad rotors are analyzed using NX 8.0 Nastran. Apart from the proposed design, the analysis holds true for all general design of quad rotor frames used worldwide.

\subsection{Displacement}

\section{Master Quadrotor Frame Analysis}

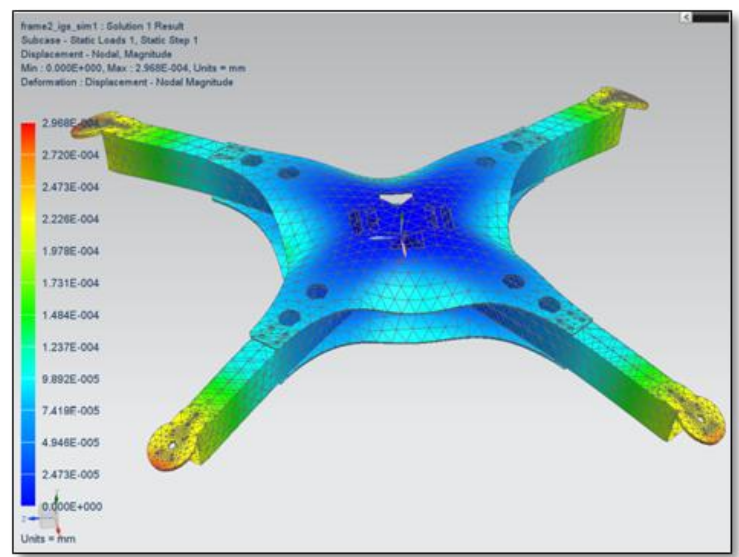

Fig. 13. Displacement in master UAV, Units: $\mathrm{mm} \mathrm{,} \mathrm{Max} \mathrm{displacement:} 0.0000296 \mathrm{~mm}$ 


\subsection{Stress}

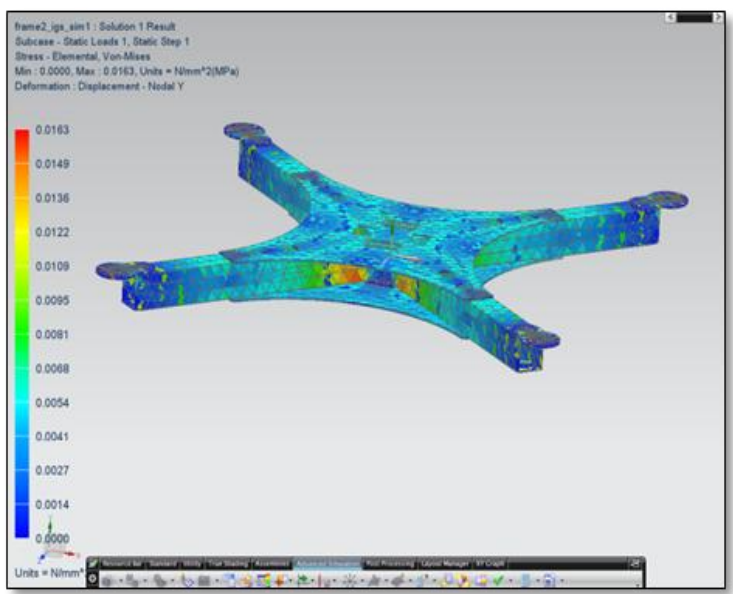

Fig. 14. Stress on UAV, Units : N/mm^2, Max stress: $0.0163 \mathrm{~N} / \mathrm{mm}^{\wedge} 2$

\subsection{Reaction Forces}

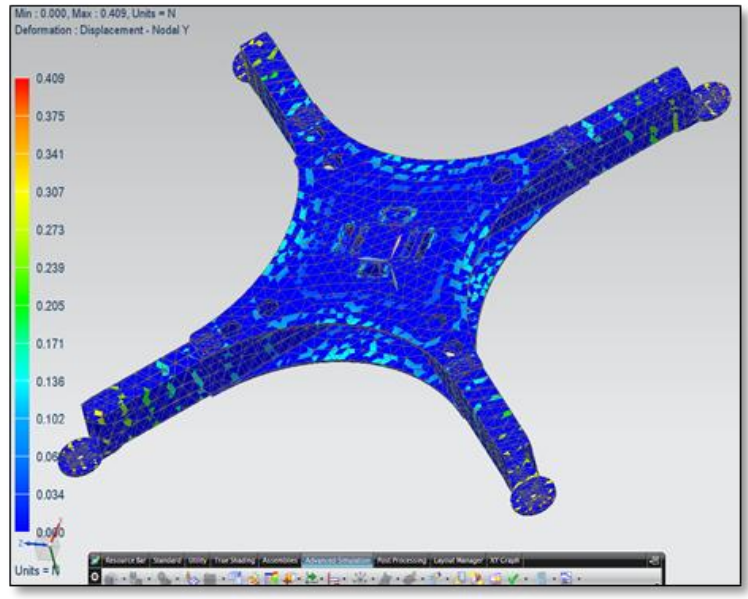

Fig. 15. Reaction Force in master UAV, Units: N Max reaction force: $0.409 \mathrm{~N}$

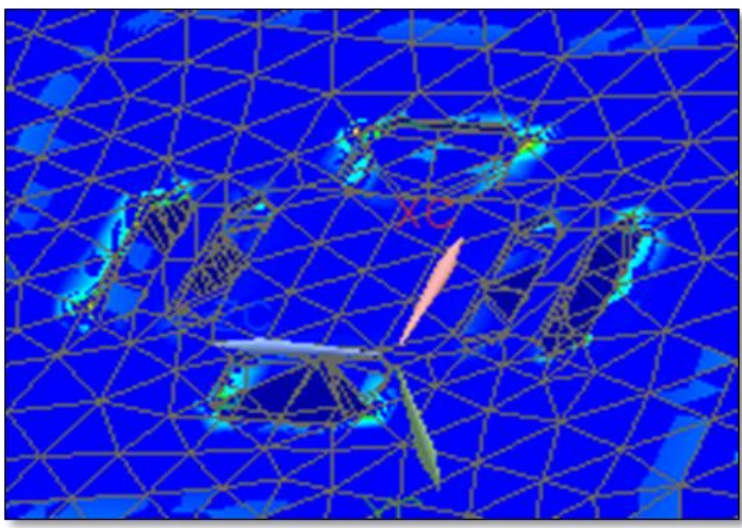

Fig. 16. Reaction at the frame locations at which the master quad-rotor is initially held before take-off.

From the above analysis of the master quad rotor design, it is evident that the design is feasible with negligible deformation. It can be concluded that maximum deformation takes place at the end of the arms, where the motors are located. 
11.1 Displacement

XI. SLAVE BI-Rotor ANALYSIS

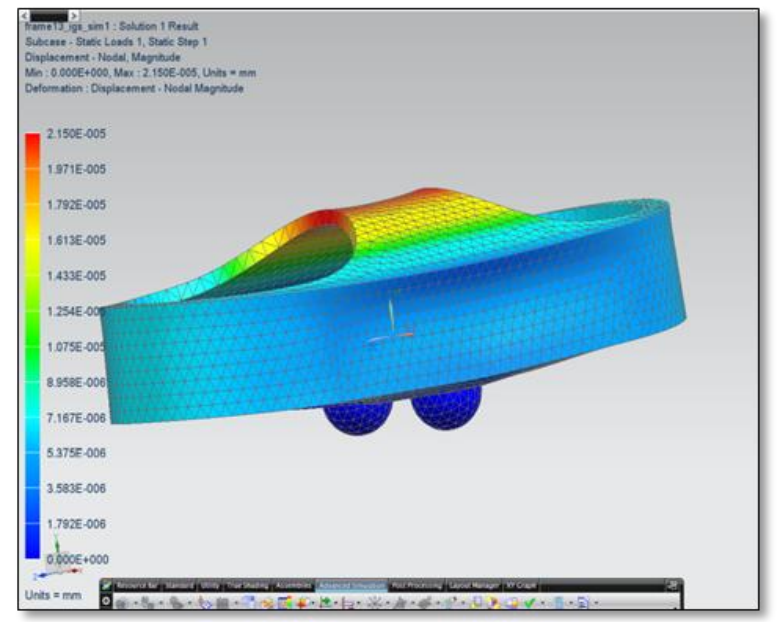

Fig. 17. Displacement in slave MAV, Units: mm Max displacement: $0.00000215 \mathrm{~mm}$

11.2 Reaction Force (When Unlatching for take-off)

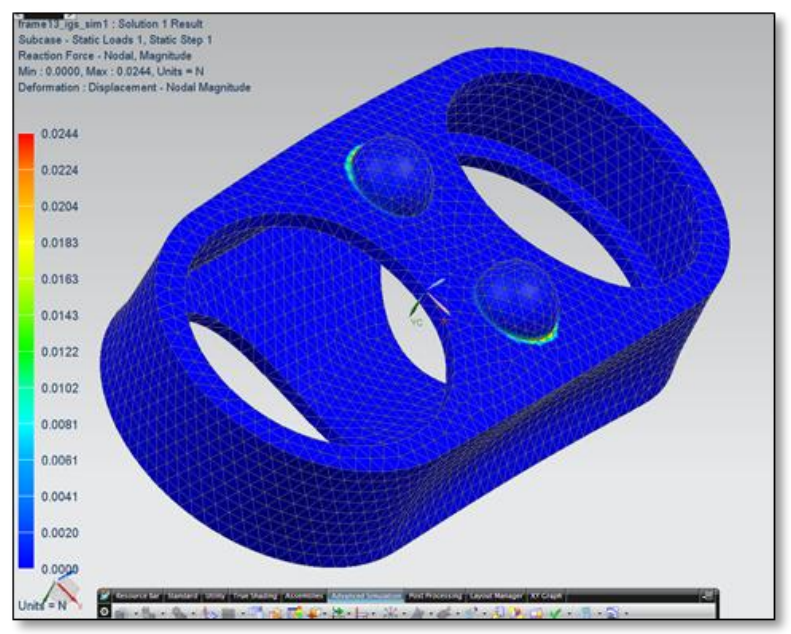

Fig. 18. Reaction Force in slave MAV, The maximum deformation takes place at the center portion of the frame Units: $\mathrm{N}$, Max reaction force: $0.0244 \mathrm{~N}$

11.3 Stress (At the Latching locations during take-off)

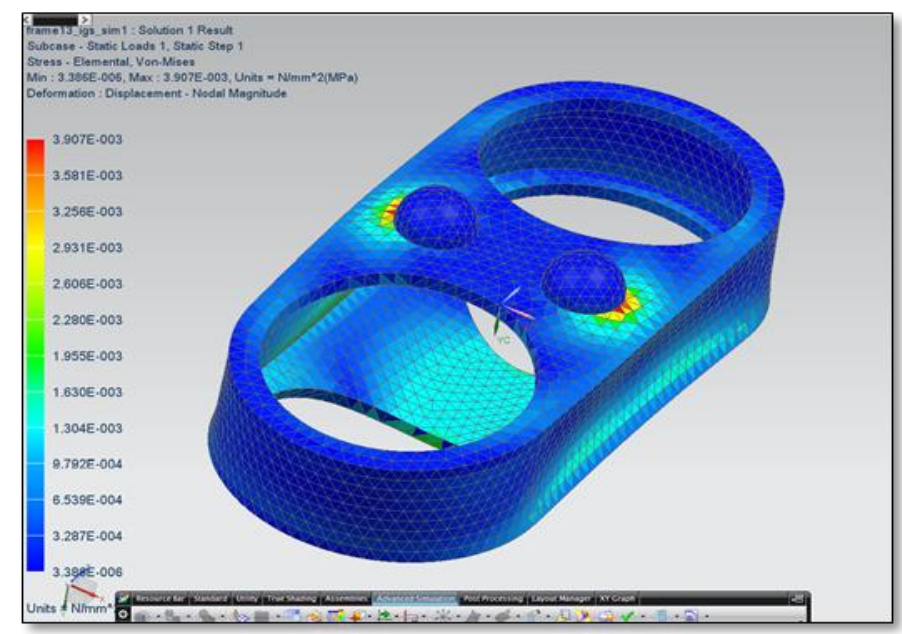

Fig. 19. Stress on MAV 


\subsection{Stress(Complete Frame)}

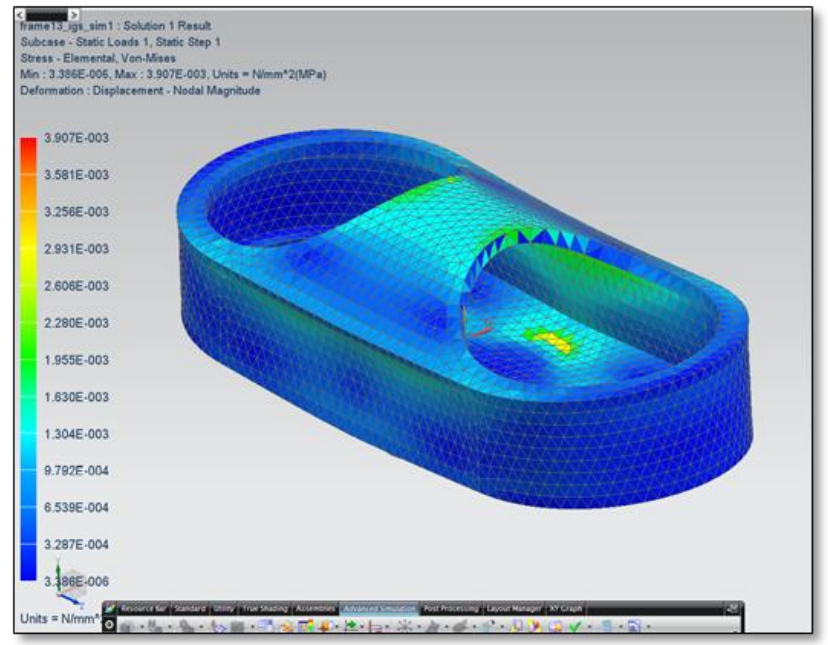

Fig. 20. Stress on MAV, Units: N/mm^2 Max stress: $0.00039 \mathrm{~N} / \mathrm{mm}^{\wedge} 2$

From the above analysis using NX 8.0 Nastran, we can conclude that the design of the slave bi-copter has negligible deformation. However the system can be augmented by increasing the material thickness at the center.

\section{CONCLUSION}

The modelled design of the frames of the master and slave vehicles are analysed for deformation, stress and reaction forces using Nastran and are found to be feasible for utilisation in fabricating the swarm controlled UAV system. The components for utilization and hardware connections of the complete system are provided. Moreover, the efficiency of the system based on the selected components is theoretically calculated and results are graphically plotted.

\section{REFERENCES}

[1] Ye Hong, Jiancheng fang, Ye Tao, Ground Control Station Development for Autonomous UAV, ICIRA '08 Proceedings of the First International Conference on Intelligent Robotics and Applications: Part II Pages 36 - 44

[2] Peter Membrey, David Hows, Learn Raspberry Pi with Linux(Apress, 2012)

[3] Carlos Francisco Escusa Palazon, Eric Arnal Fort, Oscar Casas Piedrafita, Study, Design and Construction of a Bi-Rotor And TriRotor MAV Platforms (2009)

[4] Mark A. C. Gill, Albert Y. Zomaya, Obstacle Avoidance in Multi-Robot Systems: Experiments in Parallel Genetic Algorithms (World Scientific, 1998)

[5] Panos J. Antsaklis, An Introduction to Intelligent and Autonomous Control (Boston Kluwer Academic Publishers, 1993).

[6] Petros A. Ioannou, Andreas Pitsillides, Modeling and Control of Complex Systems (CRC Press,2010)

[7] Roland Büchi , Brushless Motors and Controllers (Books on Demand, 2012)

[8] J. David Irwin, R. Mark Nelms, Basic engineering circuit analysis, 8th ed (John Wiley \& Sons, 2007)

[9] J. Gordon Leishman, Principles of Helicopter Aerodynamics (Cambridge University Press, 2006)

[10] Federal Aviation Administration(FAA), Aircraft Weight and Balance Handbook (Indomitable Publications, 2011) 